Journal of Animal and Veterinary Advances 11 (6): 731-734, 2012

ISSN: $1680-5593$

(C) Medwell Journals, 2012

\title{
Cloning and Sequence Analysis of Donkey Growth Hormone Gene
}

\author{
Wenjin Zhu, Yongmei Su and Jianhua Wu \\ Key Lab of Preventive Veterinary Medicine, College of Animal Science and Technology, \\ Hebei Normal University of Science and Technology, Hebei Province, \\ 066600 Qinhuangdao, P.R. China
}

\begin{abstract}
The PCR primers were designed online according to result of gene homology comparison. Donkey $G H$ gene DNA and cDNA sequence were cloned from liver and blood by RT-PCR and PCR, compared with GH gene sequences of different species by bioinformatics. The donkey $G H$ gene sequence was 1928 bp including the 706 bp cDNA sequence with the complete CDS. By comparison for DNA and cDNA sequences, it was found that the $G H$ gene sequence included 5 exons and 4 introns, encoding 216aa including signal protein of 26aa and matured protein of 190aa. Based on the analysis of the similarity of $G H$ genes in different species on the level of cDNA, DNA and the deduced amino acid there was the most homology to the horse. The GH gene of donkey was conservative in the process of evolution and its promotor was not specific TATA box but was CATA box. The mutation of $\mathrm{C} \rightarrow \mathrm{G}$ in 1267 may affect the growth and development of donkeys and horses. All researches made an essential foundation for $\mathrm{GH}$ regulation of gene expression, evolution, polymorphism analysis in the future.
\end{abstract}

Key words: Donkey, growth hormone gene, cloning, sequence analysis, homology, China

\section{INTRODUCTION}

Growth Hormone (GH) is a single chain polypeptide hormone secreted by the anterior pituitary, it plays an important role during animal growth and development, studies have shown that $G H$ gene consists of 5 exons and 4 introns in mammals and bird (De Noto et al., 1981; Woychik et al., 1982; Barta et al., 1991; Buggioti and Primmier, 2006). GH gene is the major gene controlling the level of GH secretion, regulating animal growth and development and other important physiological activities.

$\mathrm{GH}$ becomes one of hot spots in improving animal growth rate, growth performance and other aspects in recent years. Jorge AAM first reported the cloning of growth hormone gene sequences successfully in 1994 but study on donkey complete $G H$ gene sequence and research has not been reported before. In the present study, the complete sequence of $G H$ gene DNA and cDNA were cloned by PCR and RT-PCR and the sequence was analysised carefully in order to provide a theoretical basis for applied research.

\section{MATERIALS AND METHODS}

\section{Cloning of donkey $G H$ gene cDNA}

Total RNA extraction: The total RNA was extracted from the liver tissue of Guangling donkey based on the instruction of total RNA extracted ultra light kit. The RNA was directly used for reverse trandcription after it was detected through agarose gel electrophoresis.

PCR primer design: According to the growth hormone gene $c D N A$ coding sequence of human (M13438.1), sheep (X12546.1, X15976.1), horse (U02929), pig (U19787) and cattle (AF034386) provided by NCBI, the upstream and downstream primers of donkey $G H$ gene cDNA coding sequences was designed, respectively by Primer 3 online after multiple sequence alignment. Forward primer was 5'-TGTGGACAGCTCACCCAAC-3'; reverse primer was 5'-GCACTGAGGAGGGGTAACAG-3'.

RT-PCR test: RT-PCR was carried out based on RT-PCR kit (AMV) Ver. 3.0 instructions by using the total RNA extracted from liver as template, Oligo dT primer was used for reverse transcription and then the specific primers was designed for the PCR amplification, $3 \mu \mathrm{L}$ solution of the PCR was collected after the reaction for electrophoresis in $1 \%$ agarose. After detection, RT-PCR products were recovered and purified by rapid gel extraction kit produced by TaKaRa Biotechnolgy (Dalian) Co., Ltd. for further cloning.

Cloning and sequencing of the target gene: RT-PCR products were connected with vector pGEMR-T after

Corresponding Author: Wenjin Zhu, Key Lab of Preventive Veterinary Medicine, College of Animal Science and Technology, Hebei Normal University of Science and Technology, Hebei Province, 066600 Qinhuangdao, P.R. China 


\begin{tabular}{|c|c|c|c|}
\hline Name & Primer sequence $\left(5^{\prime} \rightarrow 3^{\prime}\right)$ & $\begin{array}{l}\text { The length of } \\
\text { amplification (bp) }\end{array}$ & $\begin{array}{l}\text { Annealing } \\
\text { temp. }\left({ }^{\circ} \mathrm{C}\right)\end{array}$ \\
\hline GH1 & $\begin{array}{l}\text { L: atgacgagcctgggggacatg } \\
\text { R: gtgcttacctgcagecatc }\end{array}$ & 267 & 68 \\
\hline $\mathrm{GH} 2$ & $\begin{array}{l}\mathrm{L}: \text { caagagaggagcgggtacag } \\
\mathrm{R} \text { : atggtctcggagaagcagaa }\end{array}$ & 940 & 69 \\
\hline GH3 & $\begin{array}{l}\text { L: ttctgcttctccgagaccat } \\
\text { R: gcactgaggaggggtaacag }\end{array}$ & 926 & 65 \\
\hline GH4 & $\begin{array}{l}\text { L: gggcagatcctcaagcaaac } \\
\text { R: tttattaggaaagatgggtaggc }\end{array}$ & 240 & 65 \\
\hline
\end{tabular}

recovered and then transformed into E. coli JM109, cultured on nutrient agar plate supplemented with AMP, IPTG and X-gal overnight. Positive colonies were picked for shaking culture and then plasmid was extracted for sequencing by Shanghai Biology Engineering Technology Ltd. (Beijing Sequencing Department) after colony PCR identification.

\section{Cloning of donkey $G H$ gene DNA}

PCR primer design: Four pairs of primer were designed according to the DNA sequence of Donkey (DQ009008), Chinese dwarf horse (DQ845298), Thoroughbred (DQ845297), pig (M17704), human (M13438.1). These primers were used to amplify different region of donkey GH gene (Table 1).

Genomic DNA extraction: The genomic DNA was extracted from the blood of Guangling donkey by applying convention method (Sambrook et al., 1999).

PCR amplification: The system of PCR was $25 \mu \mathrm{L}$, annealing temperature is shown in Table 1. Purifing and sequencing of PCR products were done by Shanghai Biology Engineering Technology Ltd.

\section{RESULTS AND DISCUSSION}

DNA and cDNA sequence of donkey $G H$ gene: The length of 1928 bp donkey GH gene was amplified by PCR using the four pair primers (Fig. 1). The length of 706 bp dinkey GH gene cDNA was obtained by PCR using the designed primer (shadow part in Fig. 1).

The sequence, $1928 \mathrm{bp}$ DNA and $706 \mathrm{bp} \mathrm{cDNA}$ of donkey $G H$ gene were compared with other animals in the NCBI database by BLAST, it was proved that it is true. The cDNA sequence is the same as 5 exons in DNA sequence, the DNA sequence included full-length of 5 exons and 4 introns. The 4 introns which lie between GT and $A G$ and are 253,214, 199 and 272 bp, respectively GT-AG is the signal of RNA splicing, it accords with GT-AG rule but the two initiation nucleotide of $5^{\prime}$ terminal is GC in pig, it do not accord with the rule (Vize and Wells, 1987).
The 5 exons which are showed in italic type (Fig. 1) and are 10,161, 117, 162 and $201 \mathrm{bp}$, respectively the length of exons in donkey $G H$ gene is the same as majority mammals. But the first exon is $13 \mathrm{bp}$ in cattle and sheep, the 3rd, 4th and 5th exon in human are 120,165 and $198 \mathrm{bp}$, respectively. Length of donkey intron is equal to horse while it is largely different with other mammals. The open reading frame of donkey $G H$ gene is $651 \mathrm{bp}$ and initiation codon is ATG, stop codon is TAG. Two flanking regions of coding region are 5'UTR and 3'UTR. Three sites before poly (A) is AATAAAA which is the signal of synthetic polyadenylate.

The protein of donkey $G H$ gene: According to Fig. 1, the open reading frame of donkey $G H$ gene is $651 \mathrm{bp}$ (23 673 bp) and encoding 216 amino acid precursor protein (Fig. 2). Precursor protein of donkey $G H$ gene was analyzed online, it showed that the molecular weight was 24471.1, isoelectric point PI was 6.83, the molecular formula was $\mathrm{C}_{1098} \mathrm{H}_{1717} \mathrm{~N}_{297} \mathrm{O}_{317} \mathrm{~S}_{10}$. Signal peptide site was analyzed by SignalP online too, the most possible shearing site lies between $26 \mathrm{aa}$ and $27 \mathrm{aa}$. The probability of signal peptide predicting is 1.0 and the probability of shearing site predicting is 0.917 .

Membrane-spanning region of the protein was analyzed by TMPRED online. It has two strong membrane-spanning region and lie 1 22aa and 100 118aa residues, respectively. Majority signal peptide sequences are included in 1 22aa residues, the membrane-spanning region mainly lies in $\mathrm{N}$-terminal through to be analyzed by TMHMM online.

The homology of donkey and other animals in DNA, cDNA and the deduced amino acid sequence: Using DNA man soft analysed the homology of DNA, cDNA and the deduced amino acid sequence of donkeys and other animals. Comparing the amino acid sequences of donkey with other animals, it suggests that precursor protein includes a signal peptide of 26aa and a mature peptide of 190aa, the result is the same as DeBao horse (Jiang et al., 2009; Yan-Ru et al., 2006).

While precursor protein of even-toed ungulate such as cattle, sheep and goat includes signal peptide of $27 \mathrm{aa}$ and a mature peptide of $190 \mathrm{aa}$ and primate includes a signal peptide of $26 \mathrm{aa}$ and a mature peptide of $190 \mathrm{aa}$. The homology of the signal peptide sequence is distinctly lower than the mature peptide sequence.

Based on Table 2, the homology between donkey and horse is the highest according to DNA, cDNA or aa sequence. Compared donkey $G H$ gene cDNA sequence with other animals, the homology successively is pig, cat, dog, cattle, sheep and goat. While the homology of aa 
ATGACGAGCCTGGGGGACATGACCCAAGAGAGGAGCGGGTACAGGATGAGTGGGAGGAGGTTCTAAATTAT CCATCAGCAGAGGCCCGTCAGTGGCCCCATGCATAAATGTATAGAGAAAATAGGTGGGGGCCGAGGGGAGA GAGAAGGAGCCTGGGCATAAAAAGGGCCCGCAGGCGACCGATTCCAGGTTCCTAGGACCCAGCTCCCCAAA CTGCTCAGGGACCTGTGGACAGCTCACCCAACTGCGATG GCTGCAGGTAAGCACCTCTAAAATCCCCCTGGG CTTGGAATCTACTGAGGGGTGGCAGGGGGCCCTGCAGATGGATGACAGCACTAACCCTGGGCTTTGGGGCTC CTGAATGTGAGCACAGACATTTGGTCAAGTTTAGAATGCTCTCAGTCCCTGTGGGAAGGAGGGGGAGGAAA GAAGTTTCCCTGAGGGAGGGAGAGGTCTGGCAGGAGACCCAGGCCTCCTGCTCTCTGCCCGCCACCCTCCAT GTGTTTCTCTAG GCCCTCGGACCTCCGTGCTCCTGGCTTTCGCCCTGCTCTGCCTGCCCTGGCCTCAGGATGTGG GCGCCTTCCCCGCCATGCCGTTGTCTAGCCTGTTTGCCAACGCTGTGCTCCGGGCCCAGCACCTGCACCAACTGGC TGCTGACACCTACAAAGAGTTT GTAAGCTTCCCAGGGATGGGTGCTCATGGAGGGTGGCAGGAAGGGGTGAAT TCCCCACCGAGGGACATAATGAGAAGAAACTGACAAGTTCAGGGTTATTTTATCCAAGCGAAGATGCTCTCT GGAGAGCATAAACTGAGGAGGGGTTCCAAAGAATCTCGGTGATGAGAACCGTGCACCAGCTTAGACCCCCG GGGGGCGTTCTTTCTCCCAGGAGCGCGCCTACATCCCCGAGGGACAGAGATACTCGATCCAGAACGCCCAGGCT GCCTTCTGCTTCTCCGAGACCATCCCGGCCCCCACGGGCAAGGATGAGGCCCAGCAGAGATCTGT]GAGTGGCCCT GCCCAGGAAGAGGGGCCTCCCTCTTTCTAAGAAGGCCGCCCTTTCGCTCCCCGGGCCCTGGGCGGCCTTGTC CCCTAGGTGGTGGAGCAGGGCAGAGGGTGTAGGGTGACGGGGTAAGGCCCTCGGGCAGAGCGGGGCCTAGA GCGGCTGCCCTGCGCCTGCGCACCCACTGCGCCCCTCCCCGCAGGACATGGAGCTGCTCCGCTTCTCGCTGCTG CTCATCCAGTCGTGGCTCGGGCCCGTGCAGTTCCTCAGCAGGGTCTTCACCAACAGCCTGGTGTTTGGCACCTCGG ACCGCGTCTATGAGAAGCTGAGGGACCTAGAGGAAGGCATCCAGGCCCTGATGCGGGTGGGGATGGCGTTGCGG GTCCCCCTCTCCTGGGTCTGGAGGCCCCTCTGGCTTAGCTGAGGGGTGGGGGCTTACATAGGCTGGGGAAGA CAGATCTCGCCGCCCTCTTTGTAGCTGTCCAGCCCTGACCTAGGAAAGATCTGACTCTTCATTTCCCCTTTTGA ATCCCCCCTGTCTTTCTCTAAGCCCGGGAAGGGAAGGTGGAAATGGAGGGGGAGGGGAGGGAGCAGCTTGC AAGTTCTCGGCCTCTCTTTTCTCCTTCTCTTCTGCAGGAGCTGGAAGACGGCAGCCCCCGGGCTGGGCAGATCC TCAAGCAAACCTACGACAAGTTTGACACAAACTTGCGCAGTGATGACGCACTGCTCAAGAACTACGGGCTACTCTCC TGCTTCAAGAAGGACCTGCACAAGGCTGAGACGTACCTGCGGGTCATGAAGTGTCGCCGCTTCGTGGAAAGCAGCT GTGCCTTCTAGTGCTGGGCTTCTCTGTTACCCCTCCTCAGTGCCTCCCTTGATCCTGGAGAGTGCCCCTCTAGT GCCTACCCATCTTTCCTAATAAAAGG

Fig. 1: The sequence of donkey $G H$ gene

MAAGPRTSVLLAFALLCLPWPQDVGAFPAMPLSSLFANAVLRAQHLHQLAADTYKEFERAYIPEGQRYSIQNAQ AAFCFSETIPAPTGKDEAQQRSDMELLRFSLLLIQSWLGPVQFLSRVFTNSLVFGTSDRVYEKLRDLEEGIQALMRE LEDGSPRAGQILKQTYDKFDTNLRSDDALLKNYGLLSCFKKDLHKAETYLRVMKCRRFVESSCAF

Fig. 2: The encoded amino acid sequence of donkey $G H$ gene

\begin{tabular}{|c|c|c|c|}
\hline Species & DNA & cDNA & $\mathrm{AA}$ \\
\hline Debao horse 67 & 98.8 & 99.5 & 99.5 \\
\hline Debao horse 46 & 98.3 & 98.9 & 98.6 \\
\hline Chinese dwarf horse & 98.5 & 99.2 & 98.1 \\
\hline Braise horse & 98.6 & 99.4 & 99.1 \\
\hline Thoroughbred & 98.4 & 99.1 & 98.6 \\
\hline Mongolian horse & 98.2 & 99.2 & 98.6 \\
\hline Cat & - & 92.8 & 98.1 \\
\hline Dog & - & 92.8 & 97.7 \\
\hline Pig & 79.4 & 94.9 & 97.2 \\
\hline Cattle & 68.3 & 91.1 & 89.8 \\
\hline Sheep & 70.9 & 90.9 & 88.9 \\
\hline Goat & - & 90.6 & 88.9 \\
\hline Human & 68.3 & 79.6 & 68.4 \\
\hline
\end{tabular}

successively is cat, dog, pig, sheep, goat and cattlt. The homology of donkey betwen primate is the lowest. This conclusion is consistent with morphological classification which prove that conservation of donkey $G H$ gene is high. The further analysis of the homology of equine $G H$ gene shows that the highest homology of aa sequence is DeBao and BaiSe horse then is Thoroughbred, Mongolian horse and it is the same trend in DNA sequence of $G H$ gene.

In the equine aa sequence, the 117 st location is Phe in donkey while it is Leu in all horse. This result is caused by the diversity of $G H$ gene DNA sequence in 1267 st site. Base of $G H$ gene DNA sequence in 1267 st site change C for $\mathrm{G}$ in equine which results in that aa change Leu for Phe in 117 st site. This may be the reason causes the growth and development difference between donkeys and horses.

Promoter of donkey $G H$ gene: Promoter is a specific DNA sequence which combines RNA polymerae and start to transcription.

Various promoters have the same or similar sequence called consensus sequence which determines the transcription activity of promoter. TATA box is a location found when researched transcription mechanism in prokaryote. In subsequent researchs, the location was discovered in majority advanced animals (Sun et al., 1990).

\section{CONCLUSION}

The 5'UTR promoter sequence of donkey $G H$ gene is predict by NNPP soft. Promoter sequence begin at the 149st and end at 199st of DNA sequence and it's length is $50 \mathrm{bp}$ (agcctgggcataaaaagggcccgcaggcgaccgattccagg 
ttcctagga). The site of predicted transcription beginning of the promoter is at 189st and it's accuracy is 1.00 . In predicted sequence, TATA frame is CATAAA (CATA box) rather than TATAAA (TATA box) in majority mammals.

This suggests that transcription mechanism in donkey is different with other animals, the combination mechanism of RNA polymerae to template is different too. If want to express a certain gene of donkey in other mammals' cells or individuals, the transcription initiation factor of donor must be removed.

\section{ACKNOWLEDGEMENTS}

This research was supported by the Natural Science Fund of Hebei province (C2007000739), Hebei Normal University of Science and Technology doctoral Start Fund (2006D006).

\section{REFERENCES}

Barta, A., R.I. Richards and J.D. Baxter, 1991. Primary structure and evaluation of rat growth hormone gene. Proc. Natl. Acad. Sci., 78: 4867-4871.

Buggioti, L. and C.R. Primmier, 2006. Molecular evolution of the avian growth hormone gene and comparison with it smammalian counterpart. J. Evol. Biol., 19: 844-854.
De Noto, F.M., D.D. Moore and H.M. Goodman, 1981. Human growth DNA sequence and mRNA structure: Possible alternative splicing. Nucleic Acids Res., 97: 3719-3730.

Jiang, Q.Y., Y.M. Wei, Y.N. Huang, X.G. Yan, G.T. Lu, X.P. Wang and H.S. Jiang, 2009. Cloning and sequencing of Growth Hormone $(\mathrm{GH})$ gene from debao dwarf horses. J. Southwest Univ. (Natl. Sci. Edn.), 12: 3135-3138.

Sambrook, J., E.F. Fritsch and T. Maniatis, 1999. Molecular Cloning: A Laboratory Manual. 2nd Edn., Cold Spring Harbor Laboratory Press, New York.

Sun, N.N., D.X. Sun and D.X. Zhu, 1990. Molecular Genetics. University of Nanjing Press, NanJing, China.

Vize, P.D. and J.R. Wells, 1987. Isolation and characterization of the porcine growth hormone gene. Gene, 55: 339-344.

Woychik, R.P., S.A. Camper, R.H. Lyons, S. Horowitz and E.C. Goodwin et al., 1982. Cloning and nucleotide sequencing of the bovine growth hormone gene. Nucleic Acids Res., 10: 7197-7210.

Yan-Ru, Z., Wunerhu and M. Lai, 2006. Cloning and sequences analysis of growth hormone gene from mongolian horse. Chinese J. Anim. Sci., 42: $10-12$. 